\title{
Development of signal generator for lab on a chip application
}

\begin{abstract}
Signal generator is an electronic device that can generate electronic signal and waveform. There are different types of signal generator available in the market such as function generator, arbitrary waveform generator, RF signal generator, vector signal generator and audio signal generator and pulse generator. Although all signal generators can generate electronic signals and waveforms, different types of signal generator are used for different applications and to develop different types of electronic signal. In this work, signal generator is designed and developed to be used in a new advance technology such as Lab- on-a-Chip (LoC) or any instrument system. It is designed by using AD9850 Direct Digital Synthesis (DDS) chip module that is able to generate sine waveform with frequency up to $30 \mathrm{MHz}$. This signal generator can accurately generate and control the waveform of various frequencies. There is limitations to the signal generator with the power supply is fixed to $5 \mathrm{~V}$. This is due to the limitation with ARDUINO UNO which is used to develop signal generator. The controlling and monitoring process on LoC always require precise control in the frequency generated in order to obtained accurate results. The ability of DDS is important to accurately control and generate waveform for various frequencies, with reliability and low cost as the main feature.
\end{abstract}

Keyword: Signal generator; DDS chip module; Waveform; Frequency 